\title{
A CATAlogue AND Finding List OF GALACTIC NOVAE*
}

\author{
H. W. DUERBECK \\ Observatorium Hoher List der Universitäts-Sternwarte Bonn, Daun, F.R.G.
}

(Received 1 August, 1983)

\begin{abstract}
A catalogue of galactic novae and an atlas of finding charts are under preparation and will be published in 1984. The status of the project is described.
\end{abstract}

The most complete lists of galactic novae been compiled by Payne-Gaposchkin (1957, 1977). These catalogues serve a statistical purpose, but lack useful information for the active observer, such as precise positions and finding charts. Major finding lists and charts were published by Khatisov (1971) and Wyckoff and Wehinger (1978) and contain 42 and 25 objects, respectively. In the former list, not all identifications are correct.

Since many novae at minimum light are within the reach of modern equipment attached to medium-size telescopes, an extended collection of nova data would now be appropriate for observers. Also, accurate positions are vital for observations in other wavelength regions, especially those with satellites.

The new catalogue and atlas of finding charts now under preparation will comprise all novae for which sufficient data are available or can be derived from a study of plate collections. It will contain:

(1) positions (equinox 1950.0 and 2000.0), accurate to at least $1^{\prime \prime}$;

(2) apparent magnitudes at maximum and minimum light;

(3) light curve types (according to the classification of Duerbeck (1981));

(4) brief notes of spectral appearances;

(5) other minimum data (e.g., orbital periods);

(6) finding charts, generally prepared from sky atlas plates.

The present status of the project is as follows: the literature search for data of 245 novae (some of them suspected ones) is essentially completed. It yielded accurate positions for $35 \%$ of the objects. For the remainder it is planned to evaluate the plates of several observatory archives with the aim of increasing the positional accuracies by factors 10 or more, as compared with previously available data. This will faciliate an identification of these novae at minimum light.

Finding charts for about $55 \%$ of the novae could be located. Most of them are insufficient due to the fact that they were intended for use during the maximum state. New standardized charts will be prepared from sky atlas plates.

The publishing data of the catalogue and atlas in 1984 .

\footnotetext{
* Paper presented at the Lembang-Bamberg IAU Colloquium No. 80 on 'Double Stars: Physical Properties and Generic Relations', held at Bandung, Indonesia, 3-7 June, 1983.
} 
For the very faint novae - e.g., in the Sgr-Sco region - which are generally poorly documented and whose minimum brightness lies in most cases below the plate limit of the sky atlases, the use of large telescopes is needed both for identifications and for further study.

Any information about unpublished identifications is welcome, also suggestions concerning the contents of the catalogue. Should, e.g., all stars once classified as novae be included even if they are now listed as other types of variable stars?

\section{Acknowledgement}

I thank the Deutsche Forschungsgemeinschaft, Bonn-Bad Godesberg, for a travel grant $(477 / 832 / 83(2))$.

\section{References}

Duerbeck, H. W.: 1981, Publ. Astron. Soc. Pacific 93, 165.

Khatisov, A. Sh.: 1971, Bull. Abastumani Astrofiz. Obs. 40.

Payne-Gaposchkin, C. H.: 1957, The Galactic Novae, North-Holland Publ. Co., Amsterdam, Holland.

Payne-Gaposchkin, C. H.: 1977, in M. Friedjung (ed.), Novae and Related Stars, D. Reidel Publ. Co., Dordrecht, Holland, p. 1.

Wyckoff, S. and Wehinger, P. A.: 1978, Publ. Astron. Soc. Pacific 90, 557. 